\title{
Análisis Primera temporada de Pesca de Anchoveta 2017
}


En abril último se inició la Primera Temporada de Pesca del Stock Norte-Centro de Anchoveta Peruana del 2017 con una cuota fijada en 2.8 millones de toneladas métricas (TM). Luego de varios años en que los desembarcos de anchoveta habían mostrado una tendencia decreciente a consecuencia de alteraciones oceanográficas esta temporada resultó bastante favorable, al menos durante sus primeras semanas.

En el presente reporte revisamos los resultados de la última temporada de pesca de anchoveta del stock norte-centro en base a información obtenida de los reportes de la actividad pesquera publicados por el Instituto del Mar del Perú (IMARPE). Así mismo, proponemos algunas medidas dirigidas a resolver problemas en la transparencia y el acceso a la información.

\section{Contexto Oceanográfico}

Es importante situar esta temporada en el contexto de cuarenta meses de alta variabilidad ambiental y en particular la presencia de El Niño durante el periodo 2015-2016.

Como se aprecia en la Figura 1, desde el 2014 se registró un fuerte impacto en los desembarcos e incluso se canceló la segunda temporada de pesca.

Posteriormente, la presencia de aguas cálidas en el sistema marino de la Corriente de Humboldt alteró la distribución y condición corporal de la mayoría de especies características de aguas frías, como en el caso de la anchoveta.

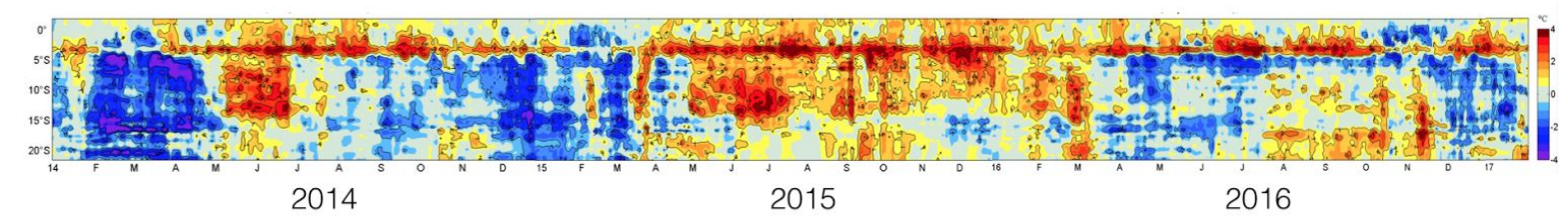

Figura 1. Diagrama Hovmöller de anomalías de temperatura superficial del mar en las primeras $\mathbf{5 0}$ millas náuticas de la costa peruana. Fuente: IMARPE

Al finalizar el año 2016 y de acuerdo a la mayoría de los modelos oceanográficos disponibles, se esperaban condiciones neutras o al menos una fase de La Niña ligera. Sin embargo, la presencia inesperada hacia mediados de febrero de un Niño Costero, que alcanzó anomalías de temperatura superficial muy alta hacia el final del mes de marzo (Fig. 2), hicieron suponer que la primera temporada de pesca sería muy mala o que podría ser cancelada.

En ese contexto se realizó la prospección Eureka LXIX entre el 21 y el 23 de febrero, durante la cual se encontró que la anchoveta presentaba una distribución continua principalmente 
superficial. Con el posterior incremento de la temperatura superficial del mar, la anchoveta se desplazó hacia el sur y a aguas más profundas en busca de ambientes favorables.

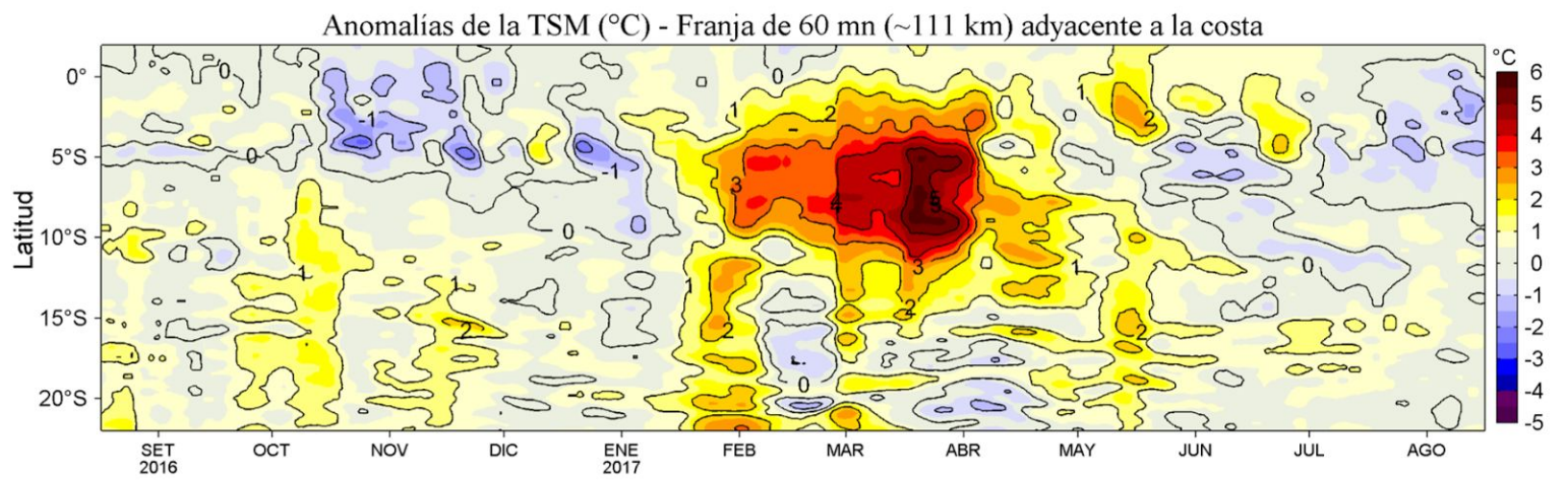

Figura 2. Diagrama Hovmöller de anomalías de temperatura superficial del mar en las primeras 60 millas náuticas de la costa peruana. Periodo Setiembre 2016 a Agosto 2017. Fuente: IMARPE Boletín Diario Oceanográfico Jueves 17 de Agosto 2017

\section{Apertura de la temporada}

Conforme se desarrollaba el Niño Costero, IMARPE realizó el Crucero 1703-04 desde el 3 de marzo al 13 de abril. La biomasa del stock norte-centro fue estimada en 7.78 millones de toneladas (LC 9.9\%), encontrándose principalmente dentro de las primeras 20 millas náuticas de la costa, con una distribución homogénea y desplazada hacia al sur como respuesta a las condiciones cálidas de el Niño Costero.

A partir de esta biomasa, IMARPE estimó una cuota de pesca tomando en consideración la necesidad de mantener una población reproductiva equivalente a 6 millones de TM al inicio de la siguiente temporada, para lo cual aplicó una tasa de explotación menor a 0.35 tal como ha sido la pauta de manejo los últimos años.

En base a esta información, el Ministerio de la Producción (PRODUCE) mediante la Resolución Ministerial RM 173-2017-PRODUCE autorizó el inicio de la primera temporada de pesca del 2017 fijando la cuota de pesca en 2.8 millones de TM. En la misma resolución se autorizó al IMARPE a realizar una pesca exploratoria del recurso entre el 22 y el 25 de abril con participación de la flota privada. Esta situación es similar a la que se ha dado en los últimos tres años y, aunque obedece a fines de conocer la distribución y condición del stock antes de iniciar la temporada; también se puede interpretar como una forma de iniciar las actividades de pesca a la brevedad posible. 
En la Figura 3 se presentan los desembarcos diarios desagregados por tipo de flota (acero naval y madera). Como se puede ver, los desembarcos fueron exitosos durante las primeras semanas de pesca y progresivamente decayeron hacia el final del mes de junio (Figura 4). Cabe señalar que algunos puertos estuvieron cerrados por varias días debido al oleaje intenso y en consecuencia, las operaciones de pesca fueron interrumpidas.

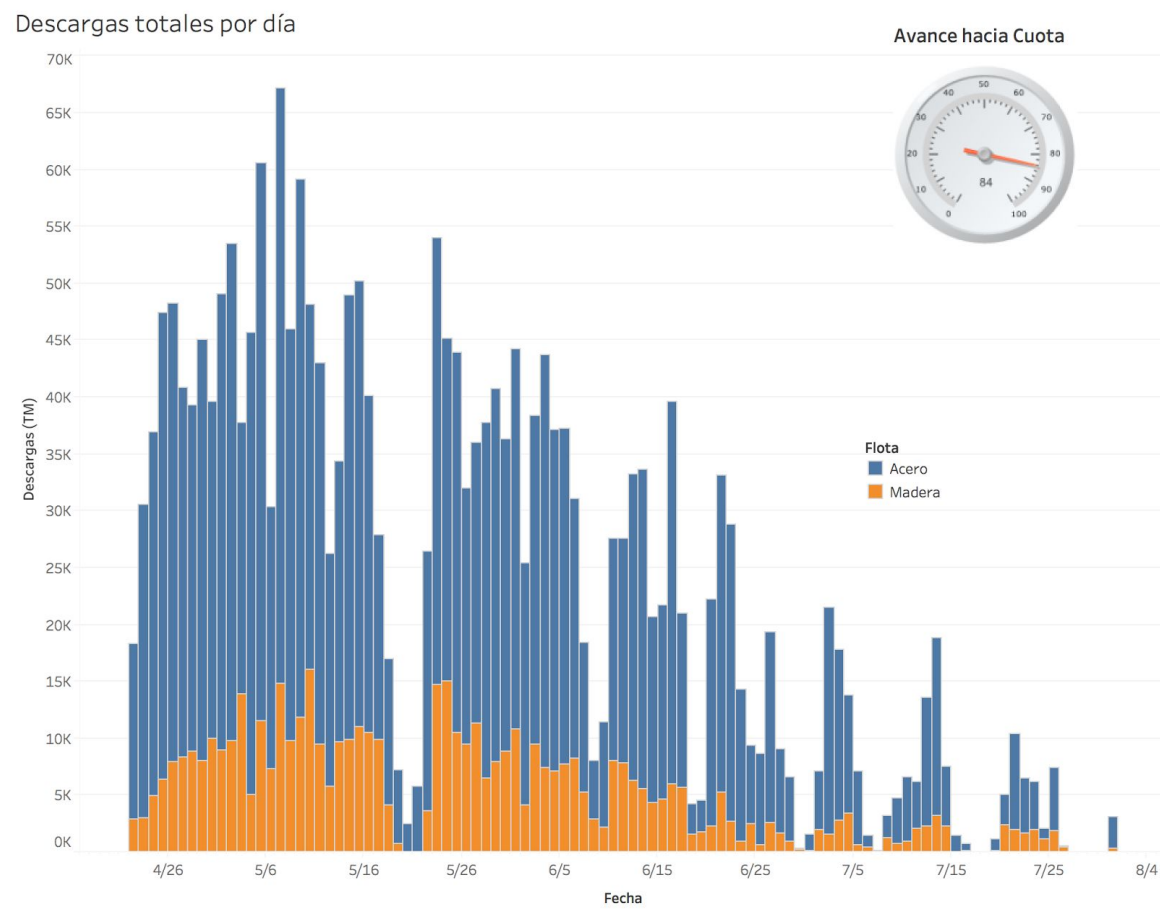

Figura 3. Descargas diarias de anchoveta por tipo de flota durante la primera temporada de pesca del Stock Norte-Centro 2017. Fuente de datos: IMARPE 




Figura 4. Descargas semanales acumuladas y promedios semanales de descargas de anchoveta durante la primera temporada de pesca del Stock Norte-Centro 2017. Fuente de datos: IMARPE

Los desembarcos promedios de ambas flotas mostraron mejor desempeño que la temporada similar del año anterior (la primera temporada del año 2016 fue cerrada tempranamente debido al inicio de la reproducción principal de invierno), pero inferiores al año 2015. Además, la flota de acero alcanzó descargas muy altas al inicio de la temporada y luego decayó, mientras que la flota de madera mantuvo descargas más modestas, pero con una menor variabilidad a lo largo de toda la temporada (Figura 5). La flota de acero habría descargado el $78.8 \%$ del total y la flota de madera el $21.2 \%$ restante. Este dato contrasta con las proporciones de años anteriores en las que la flota de acero tradicionalmente ha aportado por encima del $80 \%$ de las descargas.

De acuerdo a los datos de IMARPE, los principales puntos de desembarco de esta temporada fueron Chicama y Chimbote, que juntos suman 1.2 millones de TM descargadas, seguidos por Pisco con 0.4 millones de TM (Figura 6). 


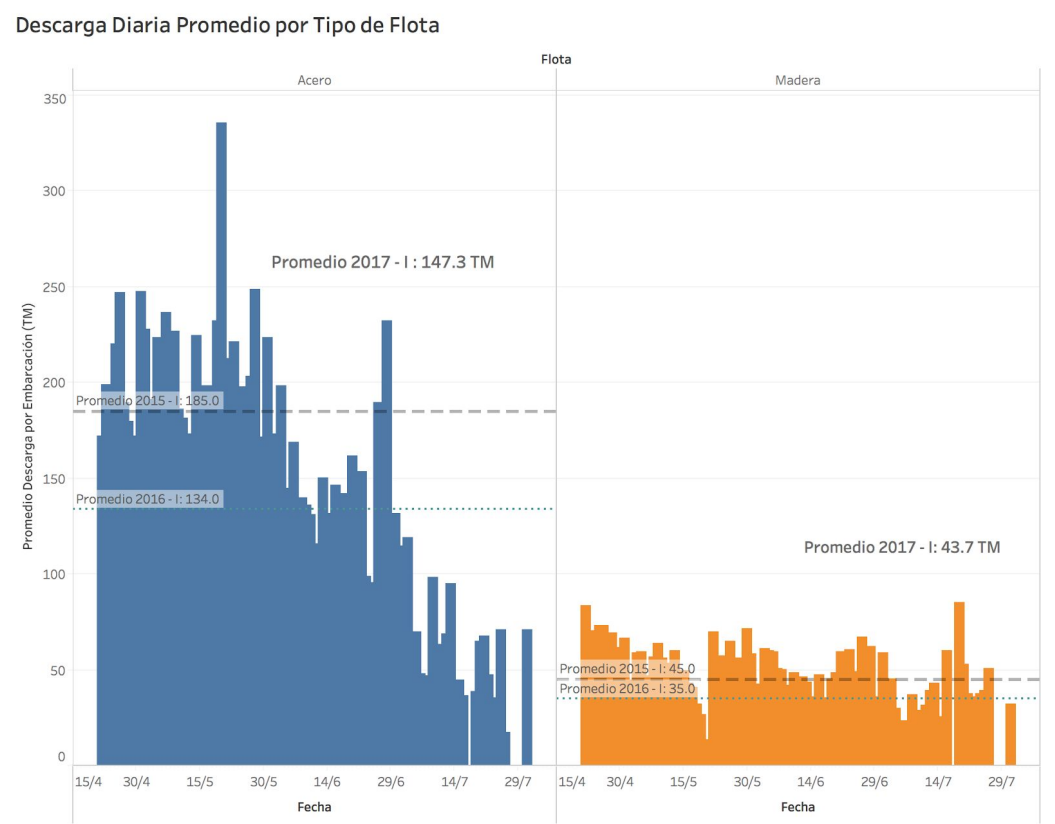

Figura 5. Descargas diarias promedio de anchoveta por tipo de flota durante la primera temporada de pesca del Stock Norte-Centro 2017. Fuente de datos: IMARPE

Descargas por Puerto



Figura 6. Descargas acumuladas de anchoveta por puerto durante la primera temporada de pesca del Stock Norte-Centro 2017. Fuente de datos: IMARPE

En comparación con las primeras temporadas de pesca de los últimos 7 años, tanto la cuota como las descargas de la primera temporada del 2017 se encuentran por encima del promedio (Figura 7). Además, aunque las descargas individuales promedio no hayan alcanzado los valores del 2010 al 2013, los desembarcos promedios diarios obtenidos de dividir el total descargado durante cada temporada entre su duración muestran un patrón ascendente (Figura 8). 


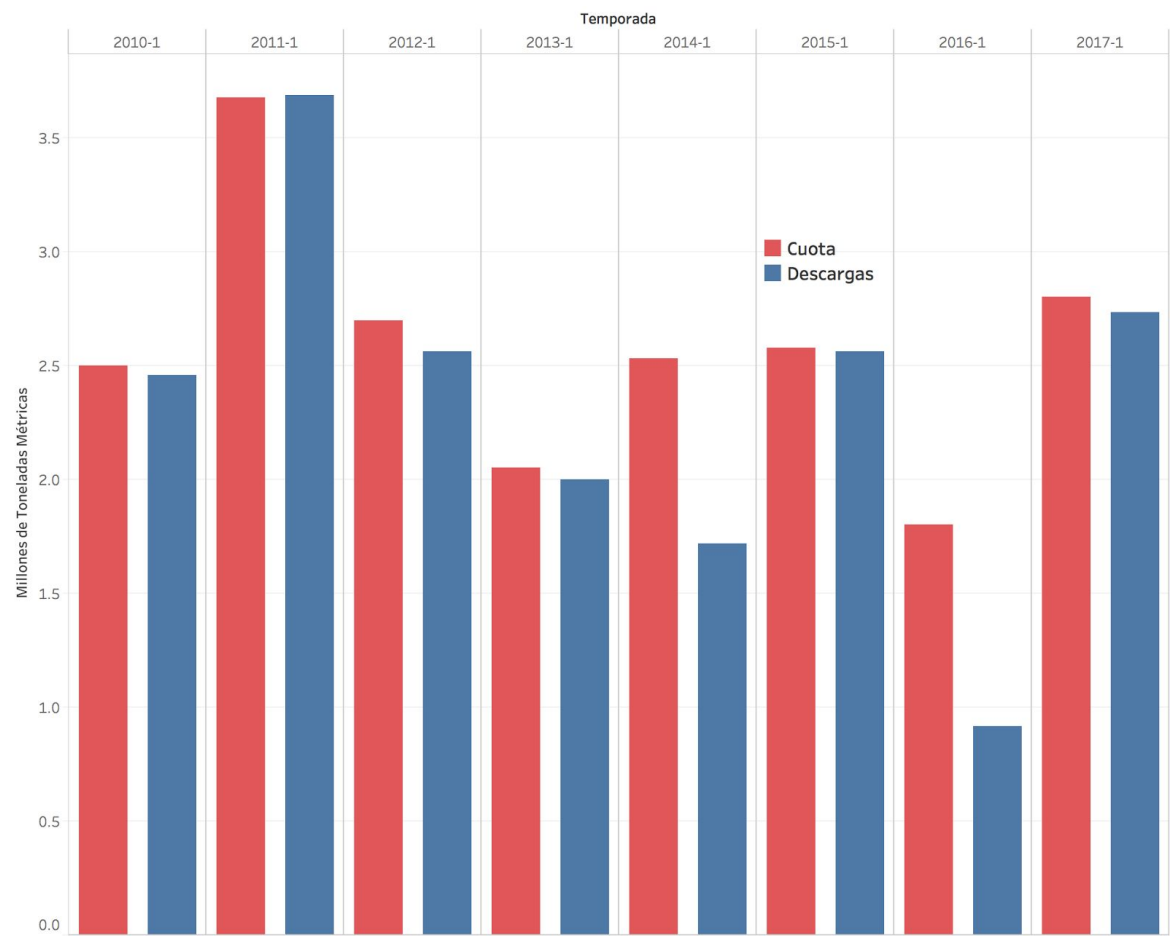

Figura 7. Cuotas de pesca y desembarques de anchoveta de las primeras temporadas de pesca para el Stock Norte-Centro. Periodo 2010 - 2017. Fuente de datos: IMARPE

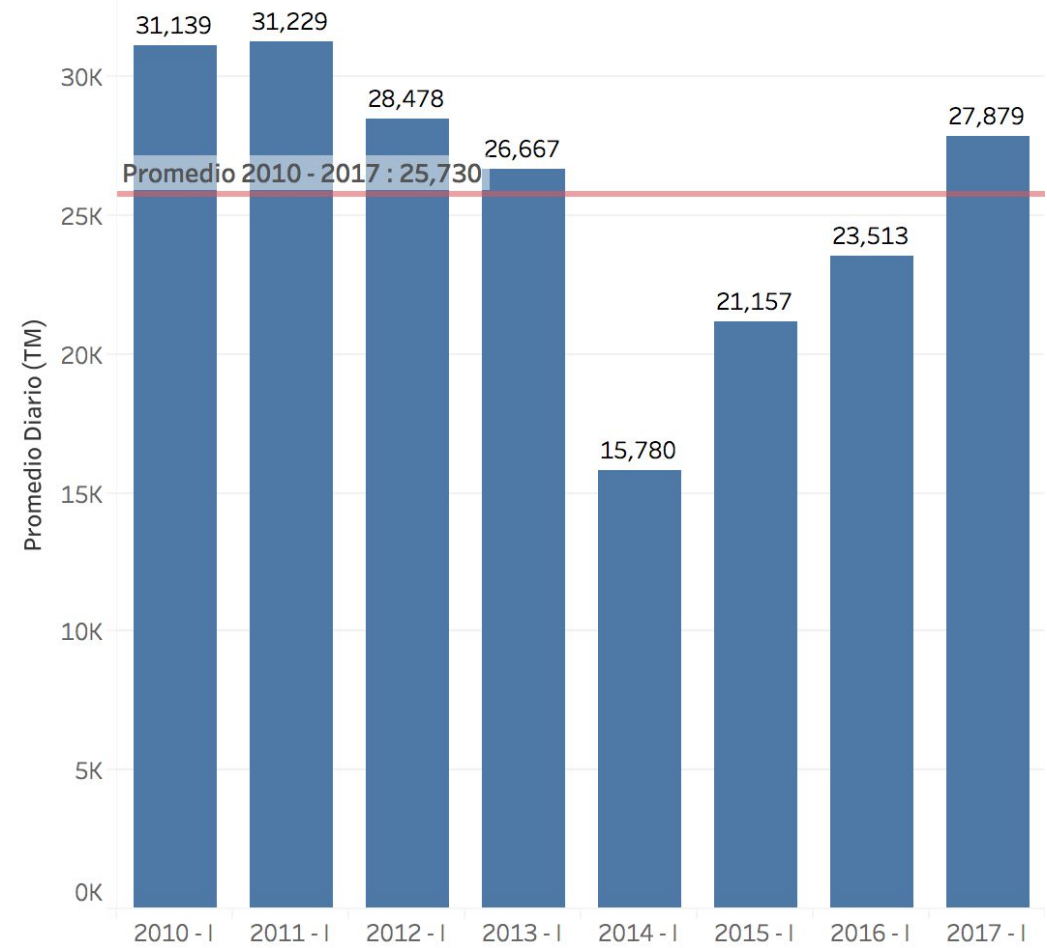

Figura 8. Desembarcos Promedio Diario de anchoveta de las primeras temporadas de pesca para el Stock Norte-Centro. Periodo 2010 - 2017. Fuente de datos: IMARPE 
La presencia de ejemplares juveniles (menores a $12 \mathrm{~cm}$ ) en las descargas fue frecuente durante el primer mes de la temporada y luego se redujo hacia el final (Figura 9). Sin embargo, algunos puertos como Tambo de Mora, Pisco, Chancay y Callao registraron varios días con frecuencias mayores al $50 \%$ de juveniles. Así también, a diferencia de la temporada anterior, los promedios diarios porcentuales de capturas de juveniles fueron menores conforme avanzó la temporada y no estuvieron correlacionados con el volumen de las descargas (Figura 10). Según datos proporcionados por PRODUCE, el total de juveniles capturado a lo largo de la temporada sería $13.96 \%$, inferior al valor tope recomendado por IMARPE en $15 \%$. Debe resaltarse sin embargo, que este límite no fue publicado en ningún documento oficial sino hasta concluida la temporada.

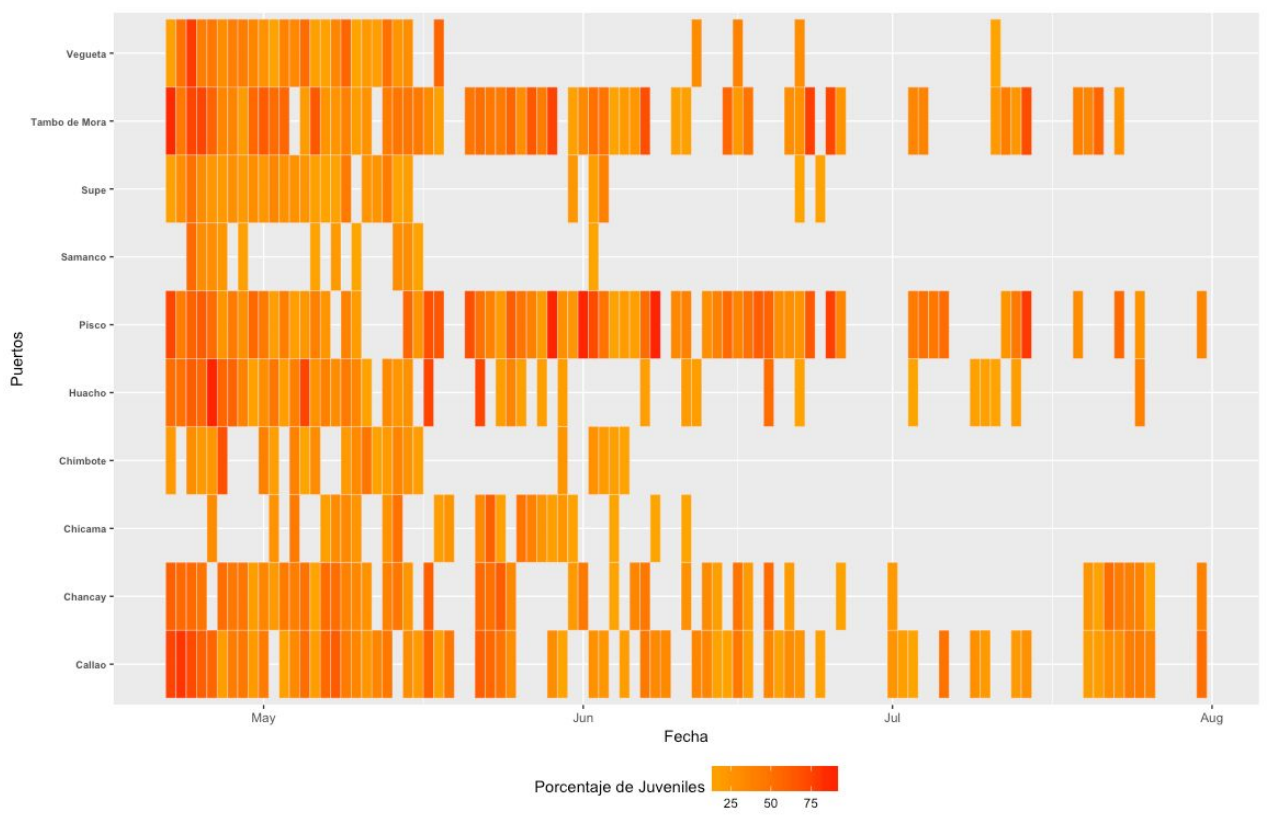

Figura 9. Frecuencia porcentual de juveniles de anchoveta durante la primera temporada de pesca del Stock Norte-Centro 2017. Fuente de datos: IMARPE 


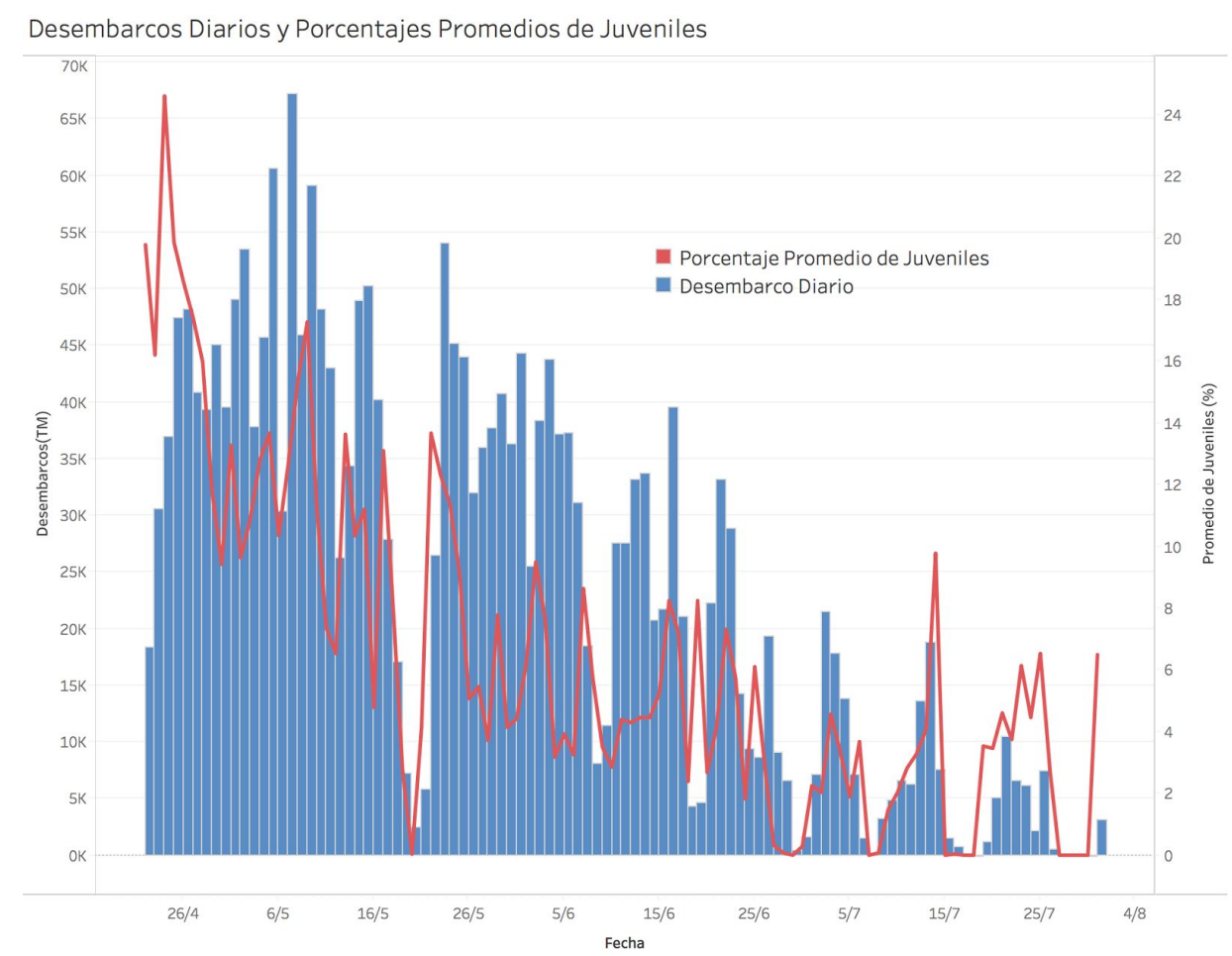

Figura 10. Desembarcos diarios y porcentajes promedios de juveniles de anchoveta durante la primera temporada de pesca del Stock Norte-Centro 2017. Fuente de datos: IMARPE

\section{Cierre de la temporada}

La temporada de pesca se cerró el 29 de julio mediante la RM 357-2017-PRODUCE, habiéndose capturado 2'72,098 TM (85.85\% de la cuota), considerando la proximidad de la temporada reproductiva de invierno y la necesidad de salvaguardar al stock. Sin embargo, tres semanas después, el índice gonadosomático estaba próximo al valor umbral (5.0), pero la actividad reproductiva medida por la fracción desovante aún estaba muy por debajo del valor crítico (27.7) (Figura 11).

Visto en perspectiva se podría especular que la temporada pudo no haber sido cerrada. No obstante, hay que considerar que, salvo la anómala primera temporada 2014, ninguna primera temporada se ha extendido después del 31 de julio bajo la consideración de preservar el stock reproductivo, lo cual constituye una saludable medida precautoria. Además, se debe tomar en cuenta que apenas se habrían descargado alrededor de 120,000 TM en las tres últimas semanas de pesca. Con capturas de anchoveta cada vez más escasa y condiciones del mar cada vez más adversas, lo más recomendable era cerrar la temporada. 

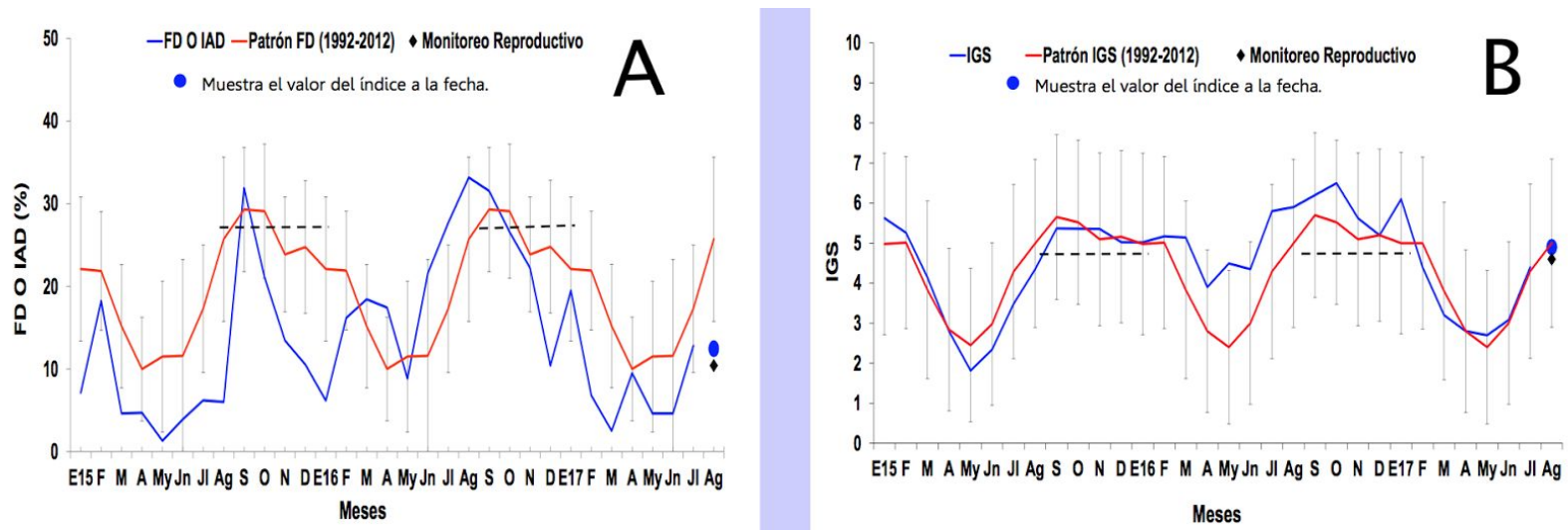

Figura 11. Fracción desovante (A) e Índice gonadosomático (B) de la anchoveta a la fecha. La línea roja muestra el patrón histórico 1992 -2012 y la línea punteada los valores críticos determinados por IMARPE. Fuente: Reporte de Indicadores Reproductivos de Anchoveta Peruana Nro 17. 14 de Agosto 2017. IMARPE.

Revisión y propuestas

En la presente temporada han subsistido algunos problemas que ya han sido identificados con anterioridad en el acceso y manejo de la información sobre todo de parte de los entes reguladores.

El principal tema es la captura incidental de juveniles, sobretodo considerando la medida dictaminada mediante el DS 024-2106, el cual permite la descarga de juveniles por encima del $10 \%$ establecido previamente, en tanto se reporte oportunamente a la autoridad. Aunque todavía es temprano para evaluar el impacto de este dispositivo en la actividad pesquera y en el manejo del stock, sería conveniente que las autoridades responsables dieran pautas claras acerca de cómo se evaluará esta política pública en el mediano plazo.

En este contexto, es importante saber en qué porcentaje se han reducido los descartes en el mar y, de otro lado, conocer, con antelación al inicio de la temporada; cuál es el valor tope (en porcentaje y volumen) de juveniles que pueden ser capturados sin comprometer la salud del stock. Reportes periódicos del avance de los valores de descarga de juveniles a lo largo de la temporada, también ayudarían a reducir las preocupaciones de los usuarios y el público.

Una situación similar se presenta con los cierres temporales que se dictaminan para proteger a ejemplares juveniles o desovantes. Esta temporada se cerraron 94 áreas de pesca con lo cual se afirma se protegieron alrededor de 758 mil TM de juveniles. Esta afirmación no tiene sustento porque los juveniles no permanecen dentro de las zonas restringidas e incluso estas solo son efectivas por pocos días con lo cual la aparente "protección" se desvanece. Esta interpretación errónea es similar a la percepción popular que las zonas cerradas son inútiles porque "en tres a cinco días los juveniles no crecen". Por ello, es preciso aclarar esta situación indicando que las zonas de cierre son temporales y como tales les dan la oportunidad a los juveniles para moverse o dispersarse a otras áreas. La medida de manejo tiene como propósito 
retrasar hasta donde sea posible su captura al reducir la presión de pesca cuando se encuentran agrupados en ciertas zonas.

Algo similar ocurre al respecto de las hembras desovantes. La anchoveta se reproduce prácticamente durante todo el año con un pico principal en invierno y otro menor en el verano (Figura 11). Por lo tanto, es importante que la autoridad invierta recursos y tiempo en informar de esta situación al público, para evitar alarma entre los usuarios del recurso y para mantener un clima de diálogo e información permanente con los gremios pesqueros.

Sobre el acceso a la información es importante señalar que IMARPE continúa siendo la principal fuente de información primaria gracias a sus reportes periódicos de la actividad pesquera. Por el lado de PRODUCE, esta temporada se suspendió la publicación de boletines periódicos, una práctica instaurada el 2016. Este año solo se produjo un documento sumario al final de la temporada. Del mismo modo, aún no hay una estructura de acceso de información centralizada sobre el avance y documentación de la temporada, tal como se estableció en la administración anterior. En general, se requiere un sistema de manejo y diseminación de información más eficiente y accesible, sobretodo para los sectores directamente involucrados en la actividad. Datos sobre descargas individuales diarias y esfuerzo de pesca serían muy útiles para tener una mejor idea del desarrollo de la temporada.

\section{Perspectiva de la segunda temporada 2017}

Como se ha señalado antes, el principal objetivo del manejo de la pesquería de anchoveta es la protección de los reproductores durante el periodo de desove y a los juveniles hasta que alcancen la talla reproductiva (reclutamiento). Protegiendo a ambos segmentos de la población (Figura 12) se crean las condiciones para que se renueve el stock en tanto las tasas de captura sean moderadas. La aplicación de estas medidas de manejo han servido para mantener la actividad pesquera a lo largo de este periodo de condiciones oceanográficas adversas para la anchoveta. 




Figura 12. Modelo conceptual de los periodos de desove y cierre de la pesquería por "veda reproductiva" adaptado de Perea et al 2011.

En la actualidad IMARPE está evaluando biomasa para lo cual emplea dos metodologías. En primer lugar, se efectuará un conteo de huevos de anchoveta, según el cual se podrán hacer proyecciones de la productividad del stock reproductivo y hacer ajustes a los modelos de dinámica poblacional. Posteriormente, se realizará el crucero hidroacústico a través del cual se mide la distribución, composición, estructura poblacional y biomasa del stock sobre el cual se fijará una cuota de pesca para la siguiente temporada.

Aunque de acuerdo a los diversos modelos climáticos, las perspectivas climáticas y oceanográficas serían positivas para la anchoveta, llama la atención el retraso en el inicio de la etapa reproductiva. De acuerdo a la información proporcionada por el último Reporte Quincenal de Indicadores Reproductivos de Anchoveta distribuido por IMARPE (Agosto 14) aún no se habrían alcanzado los umbrales para declarar el inicio oficial de la reproducción (Figura 11). Se espera que esta situación mejore, ya que en ausencia de huevos, no se podría efectuar la proyección poblacional requerida.

Por otro lado, es crucial que no se aventuren cifras sobre la cuota sin contar con los datos proporcionados por las evaluaciones científicas que realizará IMARPE. Si bien es posible desarrollar algunas proyecciones sobre la base de los modelos dinámicos de biomasa, situaciones como el retraso de la reproducción o la ausencia de reproductores, pueden modificar significativamente las especulaciones mejor intencionadas. Luego de varios años de incertidumbre y de cuestionamientos al manejo de este recurso es importante consolidar el mensaje del manejo de la anchoveta basado en evidencias científicas. 
PRODUCE, 2017. Reporte Ejecutivo de Seguimiento y Control. I Temporada de Pesca - Boletín I, 01 Ago 2017. Dirección General de Supervisión, Fiscalización y Sanción.

IMARPE, 2017. Imarpe inicia proceso de evaluación del stock norte-centro de la anchoveta que determinará inicio de la Segunda Temporada de Pesca 2017. En http://www.imarpe.gob.pe/imarpe/archivos/noticia/imarpe notic evaluacionstockanchoveta (1). pdf rev. 26/08/2017.

IMARPE, 2017. Reporte Quincenal de Indicadores Reproductivos de Anchoveta. Disponibles en http://www.imarpe.gob.pe/imarpe/lista .php?id seccion $=10146010103000000000000$

Perea Á., Peña C., Oliveros-Ramos R., Buitrón B. y Mori J. 2011. Producción potencial de huevos, reclutamiento y veda reproductiva de la anchoveta peruana (Engraulis ringens): Implicaciones en el manejo pesquero. Universidad Autónoma de Baja California, Instituto de Investigaciones Oceanológicas 37: 585-601. 